\title{
Serum Periostin Predicts Wheezing Exacerbation: A Prospective Study in Preschool Children with Recurrent Wheezing
}

\author{
Pailin Yooma Wiparat Manuyakorn Adithep Sawatchai Wanlapa Jotikasthira \\ Potjanee Kiewngam Watcharoot Kanchongkittiphon
}

Department of Pediatrics, Faculty of Medicine Ramathibodi Hospital, Mahidol University, Bangkok, Thailand

\section{Keywords}

Children · Periostin · Preschool children · Recurrent wheezing $\cdot$ Wheezing

\begin{abstract}
Introduction: Wheezing is a common problem in preschool children. Currently, there are no reliable biomarkers that can predict subsequent wheezing in preschool children. This study aimed to compare serum periostin levels between preschool children with and without recurrent wheezing and investigate its utility for predicting acute wheezing exacerbation. Methods: Children aged 2-5 years with recurrent wheezing and healthy control children were enrolled. They were evaluated for serum periostin level at enrollment and subsequently followed for wheezing episodes in a 1-year prospective study. Results: A total of 122 children were enrolled. Children in the recurrent wheezing group $(n=80)$ had a greater median serum periostin level $(1,122.32 \mathrm{pg} / \mathrm{mL}$ $[<10-6,978.93])$ than that of the healthy control group $(n=$ 40) $(<10 \mathrm{pg} / \mathrm{mL}[<10-2,116.69]), p$ value $=0.006$. After 1 -year follow-up, subjects who experienced subsequent wheezing exacerbation episodes had a greater median of periostin level $(5,321 \mathrm{pg} / \mathrm{mL})$ compared with those with no exacerbation $(<10 \mathrm{pg} / \mathrm{mL}), p$ value $=0.014$. ROC curve analysis revealed that the level of serum periostin $>1,200 \mathrm{pg} / \mathrm{mL}$, correspond-
\end{abstract}

ing to $78.9 \%$ sensitivity and $64.6 \%$ specificity, with an AUC of $0.701, p$ value $=0.009$, could be a predictor for acute wheezing exacerbation within 1 year. Besides, subjects with serum periostin $>1,200 \mathrm{pg} / \mathrm{mL}$ had greater odds of subsequent wheezing episodes compared with those with lower levels of serum periostin (adjusted odds ratio 10.0,95\% confidence interval: 2.3-43.5). Conclusions: Preschool children with recurrent wheezing have a greater serum periostin level than healthy control. Serum periostin may be a valuable biomarker for predicting acute wheezing exacerbations in the following year.

(c) 2021 The Author(s).

Published by S. Karger AG, Basel

\section{Introduction}

Wheezing disorders in children are common. About $50 \%$ of children before 6 years old have at least one wheezing episode, and up to $40 \%$ of these children develop recurrent wheezing in later childhood [1]. Children with wheezing are seen by health care providers either in the clinic or emergency department, which costs an economic burden [2]. Besides, recurrent wheezing during pre-

Edited by: D.Y. Wang, Singapore.

C 2021 The Author(s).

Published by S. Karger AG, Basel

This is an Open Access article licensed under the Creative Commons Attribution-NonCommercial-4.0 International License (CC BY-NC) (http://www.karger.com/Services/OpenAccessLicense), applicable to the online version of the article only. Usage and distribution for commercial purposes requires written permission.
Correspondence to:

Watcharoot Kanchongkittiphon, watcharoot.kan@mahidol.ac.th 
school years is one of the presenting signs of asthma [1]. Currently, there are no reliable clinical biomarkers that can predict subsequent wheezing or asthma in preschool wheezing children.

Periostin, identified as a promising biomarker involving asthma pathogenesis, is a matricellular protein whose expression can be induced by type 2 inflammatory cytokines IL-4 and IL-13 [3]. Both cytokines stimulate the secretion of periostin from lung fibroblasts [4]. Besides, IL13-stimulated epithelial cells have been shown to secrete large quantities of periostin from their basolateral aspect from bronchial epithelial cells [5], where it has pleiotropic effects on epithelial cell function and on the development of airway fibroblasts, which is thought to promote airway remodeling in asthmatic patients [5]. Periostin can also induce activation of the TGF-beta signaling pathway and increase collagen deposition, thereby contributing to airway remodeling and potentially changing its biomechanical properties [6].

Although periostin is proposed to be a favorable biomarker for type 2 inflammation of asthma pathogenesis, studies reported conflicting results. James et al. [7] measured circulating serum periostin from 1,100 subjects from the Swedish Global Allergy and Asthma European Network (GA(2)LEN) study. Periostin was positively associated with higher FENO, and an inverse relationship with lung function was observed [7]. A cross-sectional study of 120 children with asthma by El Basha et al. [8] showed that periostin levels were significantly higher during acute exacerbations, and a highly significant correlation between serum periostin level and asthma severity was found. Ionue et al. [9] found more significant serum periostin levels in 28 Japanese children with asthma than those without asthma. Furthermore, a significant cause of recurrent wheezing in preschool children is a viral infection such as respiratory syncytial virus or rhinovirus. Nakamura et al. [10] demonstrated that children with bronchitis due to respiratory syncytial virus had increased serum periostin levels. On the contrary, a study by Guvenir et al. [11] in 197 preschool children with recurrent wheezing revealed no difference in serum periostin levels between children with transient wheezing and children with asthma in both preschool and school children. A Korean study in 551 children found that serum periostin levels were not associated with allergic rhinitis or allergic sensitization [12]. As there are inconclusive data regarding an association of serum periostin level with severity of airway inflammation, particularly in children with recurrent wheezing or asthma, therefore, in the present study, we investigated the utility of serum perios- tin for predicting acute wheezing exacerbation in preschool children with recurrent wheezing.

\section{Materials and Methods}

\section{Study Design and Participants}

This is a 1-year observational prospective cohort study. After obtaining informed consent, all patients underwent an initial assessment. Participants were evaluated at a scheduled visit or via telephone contact for the episodes of doctors-diagnosed wheezing at 3, 6, and 12 months, respectively. The primary outcome of doctor-diagnosed wheezing episodes was recorded at each scheduled visit or contact. In addition, parents were asked if their child had any wheezing diagnosed by a doctor. A positive response to this question defined recurrent wheezing episodes. Children aged 2-5 years with recurrent wheezing at least 2 episodes per year confirmed by a physician [13] were enrolled in this 1-year prospective study along with healthy control subjects, followed at a well-child clinic, without any physician-diagnosed asthma or recurrent wheezing in a ratio of $2: 1$. Children with respiratory tract infection in the previous 4 weeks, inhaled corticosteroid use in the previous 4 weeks [14], underlying chronic lung disease, asthma, cardiovascular disease, autoimmune disease, malignancy, HIV infection, and immunodeficiency were excluded. Of the healthy controls, those with a history of allergen sensitization and atopy were also excluded. Baseline demographic data including age, weight, height, mode of delivery, allergic diseases, tobacco smoking exposure, parental history of allergic diseases, and history of wheezing episodes were recorded. All subjects were evaluated for serum periostin level and aeroallergens sensitization by the skin prick test (SPT) at enrollment.

\section{Skin Prick Test}

SPT was performed using a blood lancet (Feather Safety Razor, Osaka, Japan) on the volar aspect of the forearm with a positive control solution of $0.1 \%$ histamine $(1 \mathrm{mg} / \mathrm{mL})$, normal saline as a negative control, and 13 commercial extracts of aeroallergens, including Johnson grass, Bermuda grass, careless weed, Cladosporium sphaerospermum, Alternaria, Aspergillus fumigatus, Curvularia, American cockroach, German cockroach, cat hair, dog pelt, Dermatophagoides pteronyssinus, and Dermatophagoides farinae (ALK-Abello Pharm., Inc., Port Washington, NY, USA). After 15 min, a wheal size was measured, and the mean of the longest diameter plus the perpendicular diameter designated as mean wheal diameter was recorded. The size of $3 \mathrm{~mm}$ greater than the wheal size of the negative control was interpreted as positive. Atopy was defined for children with positive SPT to any of the aeroallergens $[15,16]$.

\section{Measurement of Serum Periostin}

Blood samples were collected using clot blood tubes. Serum was separated by centrifugation at 2,500 rpm for $10 \mathrm{~min}$ and then stored at $-80^{\circ} \mathrm{C}$ until the periostin level was measured. According to the manufacturer's instructions, periostin was determined by ELISA using Human Periostin/OSF-2 ELISA Kit (Abcam, UK). In brief, serum samples were added to the precoated 96-well plate with mouse-specific periostin antibody. A biotinylated detection polyclonal antibody from goat specific for periostin/OSF2 was 
Fig. 1. Flow diagram of study participants. Of all participants, 2 atopic subjects in the recurrent wheezing group refused to allow blood draw. Two subjects were lost to follow-up.

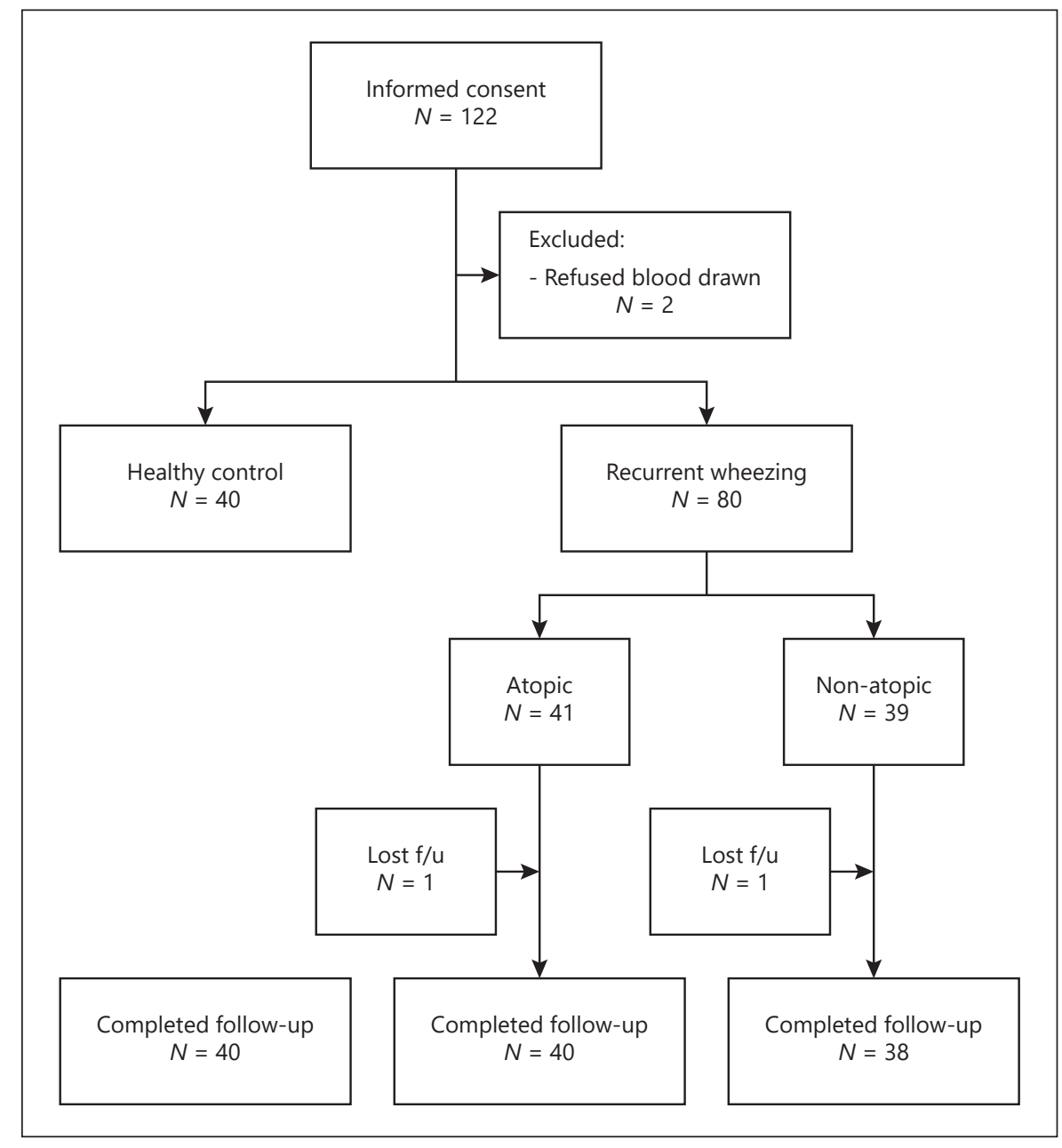

subsequently added and washed with PBS buffer. Avidin-biotinperoxidase complex was added, and unbound conjugates were washed away with PBS buffer. Tetramethylbenzidine was used to visualize horseradish peroxidase enzymatic reaction. Finally, optical density was read at $450 \mathrm{~nm}$ within $30 \mathrm{~min}$. The detection limit of this assay for assessing periostin levels was $10 \mathrm{pg} / \mathrm{mL}$. The ELISA assay was blindly performed by one of the authors.

\section{Statistical Analysis}

Statistical analysis was done using a statistical package for social sciences (SPSS version 26.0; Chicago, IL, USA). Normally distributed data were reported as mean \pm standard deviation and skewed data by median and interquartile range. Comparisons were performed by parametric (Student's $t$ test) or nonparametric (Mann-Whitney U tests) tests. The receiver operating characteristic curves were analyzed from data of children with recurrent wheezing to identify suitable periostin levels for predicting acute wheezing exacerbation and providing the optimal cutoff value. Logistic regression analysis was used for odds ratio calculation. A $p$ value of $\leq 0.05$ was considered statistically significant.

Periostin Predicts Wheezing Exacerbation in Preschool Children

\section{Results}

\section{Demographic Characteristics}

A total of 122 children aged between 2 and 5 years were classified into the recurrent wheezing group and healthy control. Two subjects were excluded due to refusal to allow blood draw (shown in Fig. 1). In the recurrent wheezing group, $45 / 80$ (54.9\%) were male, mean age 53.0 months ( \pm 11.0 months). In healthy control, $13 / 40$ (32.5\%) were male, mean age 52.4 months ( \pm 11.3 months). Subjects in the recurrent wheezing group $(n=80)$ had more parental history of allergic diseases and atopic conditions, including allergic rhinitis (40.2\%), food allergy (6.1\%), and atopic dermatitis (4.9\%), than those in the control group $(n=40)$. There were no significant differences in age, weight, mode of delivery, gestational age, and history of tobacco smoke exposure between the 2 groups ( $\mathrm{Ta}$ ble 1). 
Table 1. Baseline demographic characteristics of study participants

\begin{tabular}{llll}
\hline & $\begin{array}{l}\text { Control } \\
(n=40)\end{array}$ & $\begin{array}{l}\text { Recurrent wheezing } \\
(n=80)\end{array}$ & $p$ value \\
\hline Age, mo (SD) & $52.4(11.3)$ & $53.0(11.0)$ & 0.789 \\
Male, $n$ (\%) & $13(32.5)$ & $45(54.9)$ & $0.018^{*}$ \\
Weight, kg (SD) & $17.2(3.2)$ & $18.5(5.5)$ & 0.351 \\
Allergic comorbidities & & & $0.001^{*}$ \\
$\quad$ Allergic rhinitis, $n(\%)$ & $0(0)$ & $33(40.2)$ & 0.390 \\
$\quad$ Food allergy, $n$ (\%) & $1(2.5)$ & $5(6.1)$ & 0.157 \\
$\quad$ Atopic dermatitis, $n(\%)$ & $0(0)$ & $4(4.9)$ & 0.995 \\
Cesarean section, $n$ (\%) & $21(52.5)$ & $78(52.4)$ & 0.157 \\
Gestational age $37-42$ wk, $n(\%)$ & $40(100.0)$ & $22(14.6)$ & 0.631 \\
Tobacco smoking exposure, $n$ (\%) & $9(22.5)$ & $39(48.8)$ & $0.028^{*}$ \\
Parental history of allergic diseases & $12(30.0)$ & $3.52(0.8)$ & 0.180 \\
Mean histamine wheal size, mm (SD) & $3.66(0.7)$ & $1,122.3(<10-6,978.93)$ & $0.006^{*}$ \\
Serum periostin, pg/mL (IQR) & $<10(<10-2,116.69)$ & & \\
\hline
\end{tabular}

SD, standard deviation; IQR, interquartile range. * Statistical significance ( $p$ value $<0.05$ ).

Table 2. Differences between atopic and nonatopic recurrent wheezing participants

\begin{tabular}{|c|c|c|c|}
\hline & \multicolumn{2}{|l|}{ Recurrent wheezing } & \multirow[t]{2}{*}{$p$ value } \\
\hline & atopic $(n=41)$ & nonatopic $(n=39)$ & \\
\hline Wheezing per year, $n$, mean (SD) & $3.2(1.7)$ & $2.9(0.8)$ & 0.664 \\
\hline Wheezing apart from cold, $n(\%)$ & $34(82.9)$ & $11(26.8)$ & $<0.001^{*}$ \\
\hline Cough during playing or laughing, $n(\%)$ & $22(53.7)$ & $2(4.9)$ & $<0.001^{*}$ \\
\hline Mean histamine wheal size, $\mathrm{mm}(\mathrm{SD})$ & $3.62(0.89)$ & $3.43(0.71)$ & 0.299 \\
\hline Serum periostin, median pg/mL (IQR) & $1,122.3(<10-6,868.4)$ & $459.3(<10-7,089.4)$ & 0.421 \\
\hline
\end{tabular}

SD, standard deviation; IQR, interquartile range. * Statistical significance ( $p$ value $<0.05$ ).

\section{Baseline Serum Periostin Level}

Of all participants, children in the recurrent wheezing group had a greater median (interquartile range) serum periostin level $1,122.32 \mathrm{pg} / \mathrm{mL}[<10-6,978.93])$ than that of the healthy control group $(<10 \mathrm{pg} / \mathrm{mL}[<10-2,116.69])$, $p$ value $=0.006$ (Table 1$)$. Eighty children with recurrent wheezing were subclassified into atopic wheezing $(n=41)$ and nonatopic wheezing $(n=39)$ based on SPT sensitization. Subjects in the atopic wheezing group had more wheezing apart from cold and cough during playing or laughing than those of the nonatopic wheezing group, $p$ value $<0.01$ (Table 2). There were no significant differences in the number of previous wheezing episodes per year between groups. Children with atopic wheezing had a greater median serum periostin level $(1,122.32 \mathrm{pg} / \mathrm{mL}$ $[<10-6,868.42])$ than those of the nonatopic wheezing group $(459.31 \mathrm{pg} / \mathrm{mL}[<10-7,089.43]), p$ value $=0.421$ (Table 2).

Subsequent Wheezing Episodes during 1-Year FollowUp

After following up all participants for 1 year, 31 subsequent wheezing episodes were identified in children with recurrent wheezing. Of these, 19 wheezing episodes happened in children with atopy, whereas nonatopic children developed 12 wheezing episodes. Eleven subjects developed 1 wheezing episode, 5 subjects developed 2 wheezing episodes, 2 subjects developed 3 wheezing episodes, and 1 subject developed 4 wheezing episodes during a 1-year follow-up. None in the healthy control group developed any wheezing exacerbation episode. Two subjects (1 subject in the recurrent atopic wheezing group 


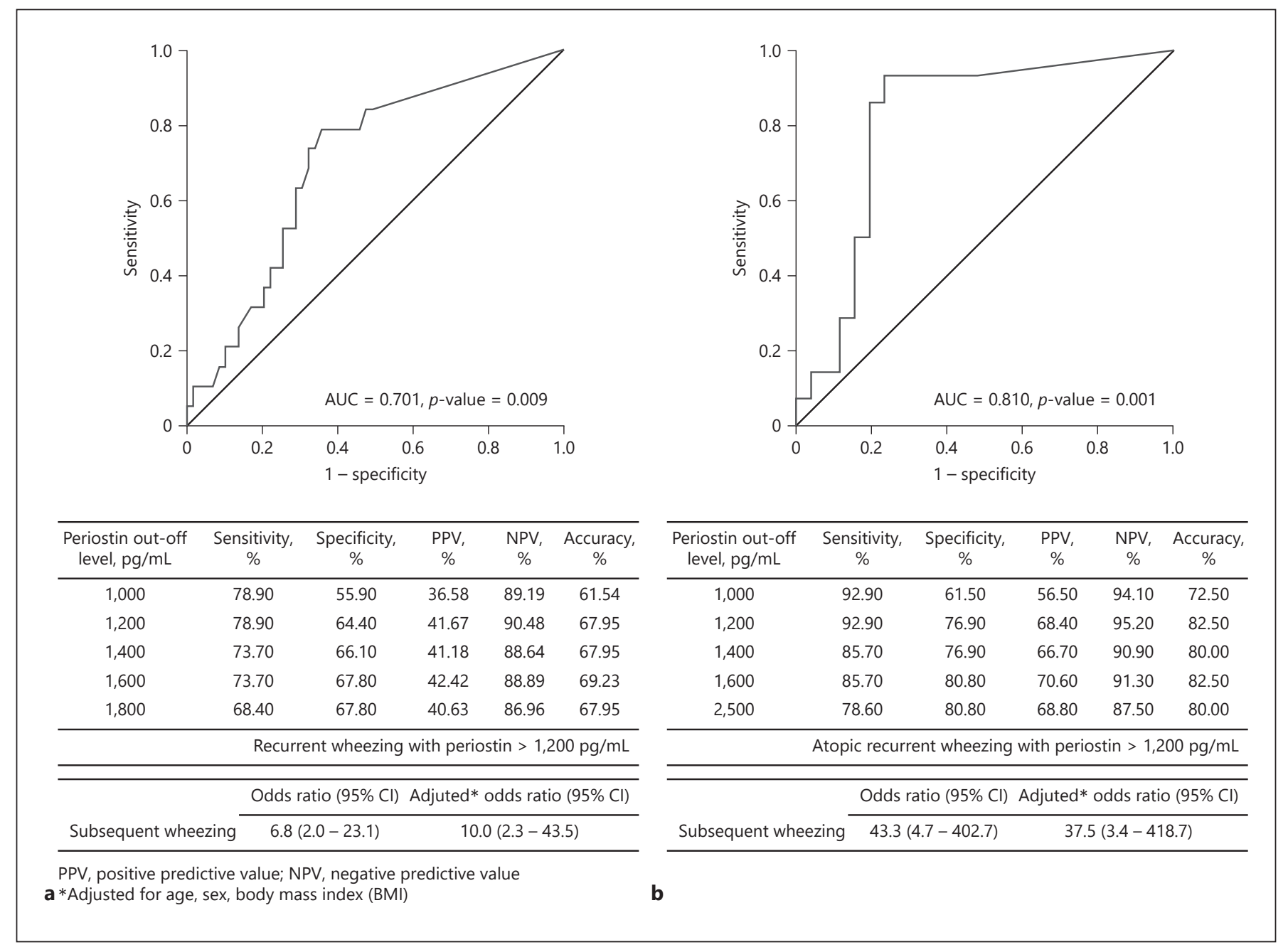

Fig. 2. ROC curves for the prediction of subsequent wheezing episodes from serum periostin level. ROC curves identified the optimal cutoff value of $1,200 \mathrm{pg} / \mathrm{mL}$ with an AUC of 0.708 for recurrent wheezing children (a) and an AUC of 0.816 for atopic recurrent wheezing children (b). The association of baseline serum

and 1 subject in the recurrent nonatopic wheezing group) were lost to follow-up (shown in Fig. 1).

Regarding subjects in the recurrent wheezing group, we found that subjects who experienced subsequent wheezing exacerbation episodes had a greater median of serum periostin level $(5,321 \mathrm{pg} / \mathrm{mL})$ compared with those with no exacerbation $(<10 \mathrm{pg} / \mathrm{mL}), p$ value $=0.014$. However, there were no significant differences in serum periostin levels between children with only 1 and $>1$ subsequent wheezing episodes, $p$ value $=0.082$. Therefore, serum periostin could be a predictor of subsequent wheezing episodes within 1 year in recurrent wheezing children.

Periostin Predicts Wheezing Exacerbation in Preschool Children periostin level $>1,200 \mathrm{pg} / \mathrm{mL}$ and subsequent wheezing episodes in the following year is shown. ROC, receiver operating characteristic; AUC, area under the curve; PPV, positive predictive value; NPV, negative predictive value. *Adjusted for age, sex, and BMI.

\section{Predicting Subsequent Wheezing Episode with Baseline Serum Periostin Level}

Receiver operating characteristic curve analysis was computed to investigate whether serum periostin level could be a predictor for subsequent wheezing exacerbation. It showed that the level of serum periostin $>1,200$ $\mathrm{pg} / \mathrm{mL}$, corresponding to $78.9 \%$ sensitivity (95\% confidence interval [CI]: 54.4-94.0) and 64.4\% specificity ( $95 \%$ CI: 50.9-76.5), with an area under the curve (AUC) of $0.701, p$ value $=0.009$, could be a predictor for acute wheezing exacerbation at 1 year in all subjects (shown in Fig. 2a). Moreover, subgroup analysis demonstrated serum periostin level $>1,200 \mathrm{pg} / \mathrm{mL}$ had $92.9 \%$ sensitivity 
(95\% CI: 66.1-99.8) and 76.9\% specificity, with an AUC of 0.816 (95\% CI: 56.1-91.0), $p$ value $=0.001$, for predicting acute wheezing episodes in atopic wheezing children (shown in Fig. 2b). Besides, subjects with serum periostin $>1,200 \mathrm{pg} / \mathrm{mL}$ had greater odds of subsequent wheezing exacerbation episodes in the following year compared with those with serum periostin $\leq 1,200 \mathrm{pg} / \mathrm{mL}$ (adjusted odds ratio [OR] 10.0, 95\% CI: 2.3-43.5) (shown in Fig. 2a). Similarly, atopic wheezing subjects with serum periostin $>1,200 \mathrm{pg} / \mathrm{mL}$ had higher odds of subsequent wheezing exacerbation episodes in the following year than those with serum periostin $\leq 1,200 \mathrm{pg} / \mathrm{mL}$ (adjusted OR 37.5, 95\% CI: 3.4-418.7) (shown in Fig. 2b).

\section{Discussion}

Our study demonstrated that serum periostin level was significantly greater in children with recurrent wheezing than healthy control children. As well, serum periostin level could be a predictor of acute wheezing exacerbation episodes of preschool children with recurrent wheezing in the following year. Despite the difference in the number of male participants in this study between children with recurrent wheezing and healthy control, Walsh et al. [17] reported that gender does not affect periostin level. Previous studies have shown that periostin levels were higher in children with asthma than in healthy controls [18-20]. Although no statistically significant difference was found, our data indicated that serum periostin levels in children with atopic wheezing tended to be greater than those of children with nonatopic wheezing. This finding was consistent with previous studies in children by Fujitani et al. [21], in which serum periostin levels in children with allergy seemed to be higher than those in nonallergic children.

Interestingly, we found that baseline serum periostin levels were greater in children with subsequent wheezing exacerbation episodes than children with no subsequent exacerbation. In agreement with this result, Scichilone et al. [22] found that serum periostin was higher in adult patients with exacerbations in the following year than those with no exacerbation. Besides, we stated that the level of serum periostin $>1,200 \mathrm{pg} / \mathrm{mL}$ could be a predictor for acute wheezing exacerbation in a subsequent year for all preschool subjects as well as subjects with atopic wheezing. Serum periostin could predict wheezing exacerbation as it has been found to prolong Th2/eosinophilic inflammation and aggravate airway remodeling [18].
We recognized several study limitations. We acknowledge the drawback of small sample size data with a potential of selection bias from a single-center study. As serum periostin was measured only at the enrollment, we could not assess intrapatient variability for periostin levels. However, low intrapatient variability of serum periostin has been demonstrated by Semprini et al. [14] and Corren et al. [23]. Regarding the level of serum periostin measurement, many studies used different assays with different reagents to measure and report different levels [7]. In this study, the reported periostin level was comparable to levels in the study by Guvenir et al. [11], also measured in preschool children. Moreover, 8 different isoforms of periostin have been characterized, which could perturb sensitivities and specificities in different assays [24]. Bone turnover and growth in children could affect periostin level, as it is the component of the extracellular matrix and regulates type I collagen formation which is essential for bone development [25]. Serum periostin levels were the highest at age 2 years, regardless of the atopic or asthmatic condition. As children grow, serum periostin was lower compared to that at the age of 2 years. However, there was no significant change in the distribution of levels between the age of 4 and 11 years [26]. Tobacco smoking exposure has been negatively associated with serum periostin levels [27]; however, there was no significant difference in tobacco smoking exposure between recurrent wheezing children and healthy children in our study. The strengths of this study are the longitudinal design and prospective follow-up with high rates of participant retention.

In conclusion, preschool children with recurrent wheezing have a greater serum periostin level than healthy control. Serum periostin level may be a valuable biomarker for predicting acute wheezing exacerbations in the following year and possibly have a role in identifying preschool children with recurrent wheezing who need early anti-inflammatory treatment. However, further studies with a longitudinal design and a bigger group of children with recurrent wheezing are needed in order to understand the exact roles of serum periostin.

\section{Acknowledgments}

We would like to thank Wasu Kamchaisatian, MD, Suwat Benjaponpitak, MD, and Cherapat Sasisakulporn for supporting and giving us valuable comments. 


\section{Statement of Ethics}

The study was conducted at Pediatric Allergy and Immunology Unit, Ramathibodi Hospital, Bangkok, Thailand, from March 2019 to December 2020. Ethical approval was provided by the $\mathrm{Hu}$ man Rights and Ethics Committee of the Faculty of Medicine, Ramathibodi Hospital, Mahidol University, Bangkok, Thailand, Approval No. MURA2019/81. Written informed consent was obtained for all participants, including their parents.

\section{Conflict of Interest Statement}

The authors have no conflicts of interest to declare.

\section{Funding Sources}

This study was supported by the Research Fund, Faculty of Medicine, Ramathibodi Hospital, Mahidol University.

\section{Author Contributions}

Pailin Yooma, MD, designed, collected data, analyzed data, wrote the manuscript, and reviewed. Wiparat Manuyakorn, MD, $\mathrm{PhD}$, designed, analyzed data, and reviewed. Adithep Sawatchai, MS, analyzed data and reviewed. Wanlapa Jotikasthira, MS, collected data and reviewed. Potjanee Kiewngam, BNS, collected data and reviewed. Watcharoot Kanchongkittiphon, $\mathrm{MD}, \mathrm{PhD}$, designed, analyzed data, drafted the manuscript, and reviewed.

\section{Data Availability Statement}

The data that support the findings of this study are not publicly available because they contain information that could compromise the privacy of research participants but are available from the corresponding author (W.K.) upon reasonable request.

\section{References}

1 Martinez FD, Wright AL, Taussig LM, Holberg CJ, Halonen M, Morgan WJ. Asthma and wheezing in the first six years of life. The Group Health Medical Associates. N Engl J Med. 1995;332(3):133-8.

2 Bui AL, Dieleman JL, Hamavid H, Birger M, Chapin A, Duber HC, et al. Spending on children's personal health care in the U S A, 1996-2013. JAMA Pediatr. 2017;171(2):1819.

3 Izuhara K, Nunomura S, Nanri Y, Ono J, Takai M, Kawaguchi A. Periostin: an emerging biomarker for allergic diseases. Allergy. 2019;74(11):2116-28.

4 Takayama G, Arima K, Kanaji T, Toda S, Tanaka H, Shoji S, et al. Periostin: a novel component of subepithelial fibrosis of bronchial asthma downstream of IL-4 and IL-13 signals. J Allergy Clin Immunol. 2006;118(1): 98-104.

5 Izuhara K, Conway SJ, Moore BB, Matsumoto $\mathrm{H}$, Holweg CT, Matthews JG, et al. Roles of periostin in respiratory disorders. Am J Respir Crit Care Med. 2016;193(9):949-56.

6 Sidhu SS, Yuan S, Innes AL, Kerr S, Woodruff PG, Hou L, et al. Roles of epithelial cell-derived periostin in TGF-beta activation, collagen production, and collagen gel elasticity in asthma. Proc Natl Acad Sci U S A. 2010; 107(32):14170-5.

7 James A, Janson C, Malinovschi A, Holweg C, Alving K, Ono J, et al. Serum periostin relates to type-2 inflammation and lung function in asthma: data from the large population-based cohort Swedish GA(2)LEN. Allergy. 2017; 72(11):1753-60.
8 El Basha NR, Osman HM, Abdelaal AA, Saed $\mathrm{SM}$, Shaaban HH. Increased expression of serum periostin and YKL40 in children with severe asthma and asthma exacerbation. J Investig Med. 2018;66(8):1102-8.

9 Inoue $\mathrm{T}$, Akashi $\mathrm{K}$, Watanabe M, Ikeda $\mathrm{Y}$, Ashizuka S, Motoki T, et al. Periostin as a biomarker for the diagnosis of pediatric asthma. Pediatr Allergy Immunol. 2016;27(5):521-6.

10 Nakamura H, Akashi K, Watanabe M, Ohta S, Ono J, Azuma Y, et al. Up-regulation of serum periostin and squamous cell carcinoma antigen levels in infants with acute bronchitis due to respiratory syncytial virus. Allergol Int. 2018;67(2):259-65.

11 Guvenir H, Buyuktiryaki B, Kulhas Celik I, Civelek E, Kilic Suloglu A, Karaaslan C, et al. Can serum periostin, YKL-40, and osteopontin levels in pre-school children with recurrent wheezing predict later development of asthma? Pediatr Allergy Immunol. 2021; 32(1):77-85.

12 Kim DY, Kim JH, Lee KH, Hong SC, Lee HS, Kang JW. Serum periostin level is not associated with allergic rhinitis or allergic sensitization in Korean children. Int J Pediatr Otorhinolaryngol. 2017;93:24-9.

13 Wi CI, Krusemark EA, Voge G, Sohn S, Liu H, Ryu E, et al. Usefulness of asthma predictive index in ascertaining asthma status of children using medical records: an explorative study. Allergy. 2018;73(6):1276-83.

14 Semprini R, Caswell-Smith R, Fingleton J, Holweg C, Matthews J, Weatherall M, et al. Longitudinal variation of serum periostin levels in adults with stable asthma. J Allergy Clin Immunol. 2017;139(5):1687-1688.e9.
15 Lawson JA, Chu LM, Rennie DC, Hagel L, Karunanayake $\mathrm{CP}$, Pahwa $\mathrm{P}$, et al. Prevalence, risk factors, and clinical outcomes of atopic and nonatopic asthma among rural children. Ann Allergy Asthma Immunol. 2017;118(3): 304-10.

16 Ronchetti R, Jesenak M, Rennerova Z, Barreto M, Ronchetti F, Villa MP. Relationship between atopic asthma and the population prevalence rates for asthma or atopy in children: atopic and nonatopic asthma in epidemiology. Allergy Asthma Proc. 2009;30(1):55-63.

17 Walsh JS, Gossiel F, Scott JR, Paggiosi MA, Eastell R. Effect of age and gender on serum periostin: relationship to cortical measures, bone turnover and hormones. Bone. 2017;99: 8-13.

18 Li W, Gao P, Zhi Y, Xu W, Wu Y, Yin J, et al. Periostin: its role in asthma and its potential as a diagnostic or therapeutic target. Respir Res. 2015;16(1):57.

19 Lopez-Guisa JM, Powers C, File D, Cochrane E, Jimenez N, Debley JS. Airway epithelial cells from asthmatic children differentially express proremodeling factors. J Allergy Clin Immunol. 2012;129(4):990-7.e6.

20 Song JS, You JS, Jeong SI, Yang S, Hwang IT, Im YG, et al. Serum periostin levels correlate with airway hyper-responsiveness to methacholine and mannitol in children with asthma. Allergy. 2015;70(6):674-81.

21 Fujitani H, Kasuga S, Ishihara T, Higa Y, Fujikawa S, Ohta N, et al. Age-related changes in serum periostin level in allergic and non-allergic children. Allergol Int. 2019;68(2):2856. 
22 Scichilone N, Crimi C, Benfante A, Battaglia S, Iemmolo M, Spatafora M, et al. Higher serum levels of periostin and the risk of exacerbations in moderate asthmatics. Asthma Res Pract. 2016;2(1):1.

23 Corren J, Lemanske RF, Hanania NA, Korenblat PE, Parsey MV, Arron JR, et al. Lebrikizumab treatment in adults with asthma. N Engl J Med. 2011;365(12):1088-98.
24 Bai Y, Nakamura M, Zhou G, Li Y, Liu Z, Ozaki T, et al. Novel isoforms of periostin expressed in the human thyroid. Jpn Clin Med. 2010;1:13-20.

25 Kudo A. Periostin in fibrillogenesis for tissue regeneration: periostin actions inside and outside the cell. Cell Mol Life Sci. 2011;68(19): 3201-7.
26 Anderson HM, Lemanske RF Jr, Arron JR, Holweg CTJ, Rajamanickam V, Gangnon RE, et al. Relationships among aeroallergen sensitization, peripheral blood eosinophils, and periostin in pediatric asthma development. J Allergy Clin Immunol. 2017;139(3):790-6.

27 Yon DK, An J, Ha EK, Jee HM, Izuhara K, Ono J, et al. Serum periostin is negatively correlated with exposure to formaldehyde and volatile organic compounds in children. $\mathrm{Al}-$ lergy Asthma Immunol Res. 2018;10(6):71621. 